\title{
BAF53a is a potential prognostic biomarker and promotes invasion and epithelial-mesenchymal transition of glioma cells
}

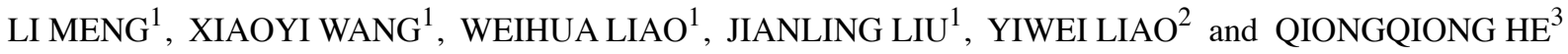 \\ Departments of ${ }^{1}$ Radiology, ${ }^{2}$ Neurosurgery and ${ }^{3}$ Pathology, Xiangya Hospital, \\ Central South University, Changsha, Hunan 410008, P.R. China
}

Received April 7, 2017; Accepted September 25, 2017

DOI: $10.3892 /$ or.2017.6019

\begin{abstract}
Increasing evidence indicates that BAF53a is crucial for embryonic development and maintenance of stemness, and may be associated with epithelial-mesenchymal transition (EMT), which suggests its involvement in cancer progression. However, the role of BAF53a in glioma remains unknown. In the present study, BAF53a was found to be highly expressed in glioma tissues and was associated with poor overall survival (OS) and progression-free survival (PFS) in glioma patients. A multivariate Cox regression analysis revealed that BAF53a might be an independent prognostic factor for OS and PFS in glioma patients. Further functional analysis indicated that BAF53a overexpression could promote proliferation and increase the motility and invasion of U87 glioma cells, whereas BAF53a knockdown had the opposite effect. In addition, BAF53a expression was associated with the levels of E-cadherin and vimentin expression in glioma tissues. This was further confirmed in U87 cells expressing different levels of BAF53a; BAF53a overexpression was concomitant with decreased $\mathrm{E}$-cadherin and increased vimentin expression, whereas BAF53a knockdown showed the opposite pattern of expression. Taken together, these results suggest that BAF53a may be a novel prognostic factor for glioma patients, and that BAF53 may facilitate glioma progression by promoting proliferation, invasion, and associate with EMT. Therefore, BAF53a could be a potential promising biomarker and a target for the treatment of glioma.
\end{abstract}

\section{Introduction}

Glioma is among the most common and lethal malignant brain tumors in humans (1). Despite advancements in surgical, radiotherapeutic and chemotherapeutic treatments, the survival

Correspondence to: Dr Xiaoyi Wang, Department of Radiology, Xiangya Hospital, Central South University, Xiangya Road 87, Changsha, Hunan 410008, P.R. China

E-mail: echo96130@126.com

Key words: glioma, BAF53a, epithelial-mesenchymal transition, tumor progression, prognosis of glioma patients remains very poor, and the majority of patients die within 2 years of diagnosis due to cancer progression (2). According to the World Health Organization (WHO) grading criteria, glioma is classified into four grades (I-IV), and survival rate has been reported to worsen with increasing grade; the 5-year survival rate is $30-70 \%$ in patients with grade I/II, compared with $3 \%$ in patients with grade IV disease (3). However, the detailed mechanisms underlying these differences in survival rates between different tumor grades remain obscure. Therefore, it is necessary to better understand the molecular mechanisms of glioma progression and thus develop novel therapeutic strategies.

Recently, studies have suggested that the epithelial-mesenchymal transition (EMT) plays an important role in cancer progression $(4,5)$. EMT is the biological process in which cells are converted from an epithelial phenotype into a mesenchymal-like phenotype, which is involved in development and tissue regeneration. The hallmarks of EMT are decreased expression of epithelial markers (E-cadherin, $\beta$-catenin, etc.) and increased expression of mesenchymal markers (vimentin, $\mathrm{N}$-cadherin, etc.), as well as changes in cell morphology (6). Numerous studies have confirmed that EMT is involved in the invasion and metastasis of cancers, including glioma. Patients with glioma that exhibit EMT-related changes typically have decreased survival times, and glioma cells that undergo EMT have stronger migratory and invasive potentials (7-9). Hence, further exploration of the association between EMT and glioma is critical for clarifying the mechanism of glioma progression.

BAF53a (also known as ACTL6A or ARP4) is a subunit of the $\mathrm{Brg} / \mathrm{Brm}$-associated factor (BAF) complex, which is crucial for embryonic development and maintenance of stemness in stem or progenitor cells (10-12). Therefore, BAF53a may also be associated with EMT. Recently, studies have reported that the aberrant expression of BAF53a is correlated with the progression of rhabdomyosarcoma, osteosarcoma, hepatocellular carcinoma and head and neck squamous cell carcinoma (HNSCC) (13-16). Based on its biological function in stem and progenitor cells, BAF53a is considered to promote cancer progression via EMT. However, the role of BAF53a in the invasion and metastasis of glioma remains unclear to date.

In the present study, we report for the first time that a high level of BAF53a expression in glioma specimens is associated with poor prognosis, and that the overexpression of BAF53a 
can promote the proliferation and invasion of glioma cells. Furthermore, BAF53a expression was found to be significantly associated with the expression of EMT markers, which indicates that BAF53a may promote the metastasis of glioma through EMT. Taken together, these findings preliminarily indicate that BAF53a may be a novel prognostic marker and promising therapeutic target for glioma.

\section{Materials and methods}

Patient specimens and cell cultures. The 121 glioma tissue samples were randomly obtained from the Department of tissue, Xiangya Hospital between January 2009 and December 2012. All the gliomas were pathology confirmed by 2 independent pathologists according to the 2007 WHO classification. All patients eligible for this study acceptted surgery and were regularly followed-up, and collected detailed clinicopathological data and survival data. Magnetic resonance imaging (MRI) or contrast-enhanced MRI was performed every 6 months. OS was defined as the time from the surgery to the death or the last follow-up visit. PFS was defined as the time from the surgery to the first evidence of recurrence, progression, or death. All patients signed a written informed consent. The study was approved by the ethics committee of Xiangya Hospital in accordance with the Declaration of Helsinki.

The human primary glioma cell line U87 was obtained from the Type Culture Collection of the Chinese Academy of Sciences (Shanghai, China), and cultured in DMEM (Gibco, NY, USA) with $10 \%$ fetal bovine serum (Gibco) at $37^{\circ} \mathrm{C}$ in a humidified atmosphere of $5 \% \mathrm{CO}_{2}$.

RNA isolation and real-time PCR. Total RNA of glioma tissues and cells were isolated with TRIzol reagent (Invitrogen, CA, USA) following the manufacturer's instructions. The concentration and purity of all RNA samples were determined by NanoDrop Spectrophotometer (NanoDrop Technologies, TX, USA). Then, the cDNA was synthesized by reverse transcription of total RNA using cDNA synthesis kit (Roche Life Sciences, Switzerland), and real-time PCR reaction was conducted using the SYBR Green PCR kit (Roche Life Sciences) and performed on ABI PRISM 7100 Sequence Detection system (Applied Biosystems, CA, USA) following the manufacturer's instructions. GAPDH served as control. The target mRNA expression was calculated using the $2^{-\Delta \Delta C t}$ method. Each experiment was repeated 3 times. The primers were as follows: BAF53a (forward, 5'-CCAGGTCTCTAT GGCAGTGTAA-3'; reverse, 5'-CGTAAGGTGACAAA AGGAAGGTA-3'); GAPDH (forward, 5'-GTCTCCTCTGAC TTCAACAGCG-3'; reverse, 5'-ACCACCCTGTTGCTGTAG CCAA-3'); E-cadherin (forward, 5'-GCCTCCTGAAAAGAG AGTGGAAG-3'; reverse, 5'-TGGCAGTGTCTCTCCAAAT CCG-3'); vimentin (forward, 5'-AGGCAAAGCAGGAGTCCA CTGA-3'; reverse, 5'-ATCTGGCGTTCCAGGGACTCAT-3').

Protein extraction and western blotting. Fresh tissues or cells were dissolved by RIPA lysis buffer (Invitrogen) and PMSF (Zhongshan Goldenbridge Biotechnology, Shanghai, China). Then isolated by centrifugation and quantified by Bradford Protein assay kit (Beyotime Institute of Biotechnology, Shanghai, China). Next the protein was separated by
10\% SDS-PAGE and transferred to the PVDF membrane (Sigma, MO, USA). The membranes were blocked with $5 \%$ calf serum and incubated with appropriate primary antibody, followed by incubation with secondary antibody. Lastly, the signal was detected using enhanced chemiluminescence regents (Thermo Scientific, MA, USA) and photographed by camera and image processing system (Bio-Rad, CA, USA).

Immunohistochemistry. The glioma tissues were embedded in paraffin, and sectioned into slides with a thickness of $4 \mu \mathrm{m}$. Then, sections were dewaxed in xylene and rehydrated in graded ethanol. Antigen retrieval was accomplished using citrate buffer at $\mathrm{pH}$ 6.0. Then $3 \% \mathrm{H}_{2} \mathrm{O}_{2}$ was used to block endogenous peroxidase activity, and goat serum incubation was used to block the non-specific antigen binding, followed by incubation with appropriate concentration of primary antibody at $4^{\circ} \mathrm{C}$ overnight. Then, the sections were incubated with the corresponding secondary antibody for $30 \mathrm{~min}$ at room temperature after rinsing three times in PBS. The antigen-antibody interactions was detected by DAB and counterstained by hematoxylin, followed by dehydrated in graded ethanols and mounted. The results were evaluated by two independent pathologists blinded to the study. The staining intensity of BAF53a was scored according to the percentage of positive stained tumor cells, as negative $(-)<5 \%$ of tumor cells stained positive, (+) $0-25 \%,(++) 26-75 \%,>76 \%$ of tumor cells stained positive.

Lentiviral vectors production and cellular transduction. Full-length human BAF53a overexpression clone lentivirus and short hairpin RNAs (shRNA) lentivirus and their control vectors were constructed by a biotechnology company (GeneChem, Shanghai, China). Cells were cultured in 6-well plates before transfection until 80-90\% confluency within $24 \mathrm{~h}$. Then the lentivirus were transfected into cells according to the manufacturer's instructions. Puromycin $(2 \mu \mathrm{g} / \mathrm{ml})$ was used to select stable clones if necessary. After 48-72-h transfection, the cells were harvested for subsequent assays. Transfection efficiency was assessed by fluorescence microscope, real-time PCR and western blotting.

Reagents. Mitomycin-C and puromycin were purchased from ApexBio, Technology LLC A4452, A3740; ApexBio, TX, USA). The Matrigel matrix was purchased from Corning Life Sciences (354248; Corning, MA, USA). The primary antibodies against BAF53a (sc-137062), E-cadherin (sc-71007), vimentin (sc-80975), and $\beta$-actin (sc-130300) were purchased from Santa Cruz Biotechnology (CA, USA).

Proliferation assays. The proliferation potential was measured by MTT assay and colony formation assay. For MTT assays, the cells were grown into 96-well plates with a density of $5 \times 10^{3}$ cells/well. Each day 6 wells of each group were added $100 \mu 1$ MTT $(0.5 \mathrm{mg} / \mathrm{ml}$; Sigma) reagent per well and incubated at $37^{\circ} \mathrm{C}$ for $4 \mathrm{~h}$. Subsequently, the absorbance values were measured at $570 \mathrm{~nm}$. For colony formation assays, $2 \times 10^{3}$ cells/well were seeded into 6-well plates and cultured for 14 days. Then the cell colonies were dyed with crystal violet and counted. The results were recorded as mean \pm SD of three repeats. 
A

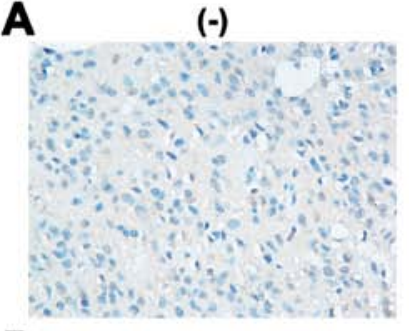

B
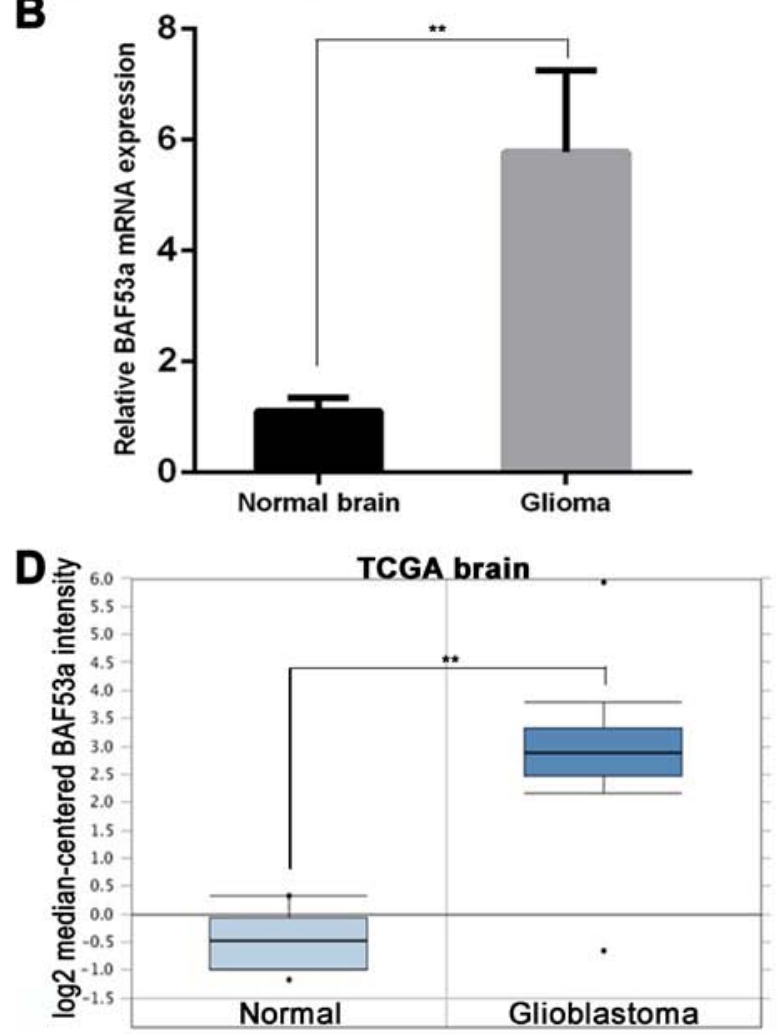

$(+)$

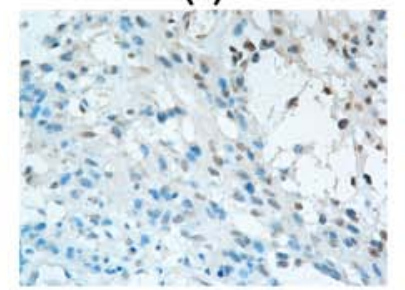

c

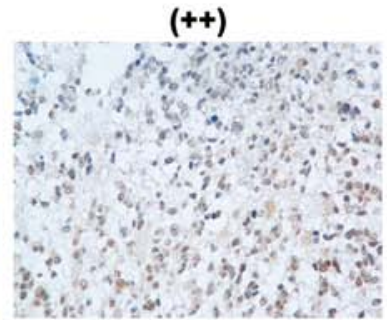

$(+++)$

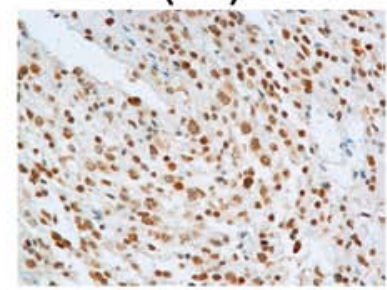

Sun brain

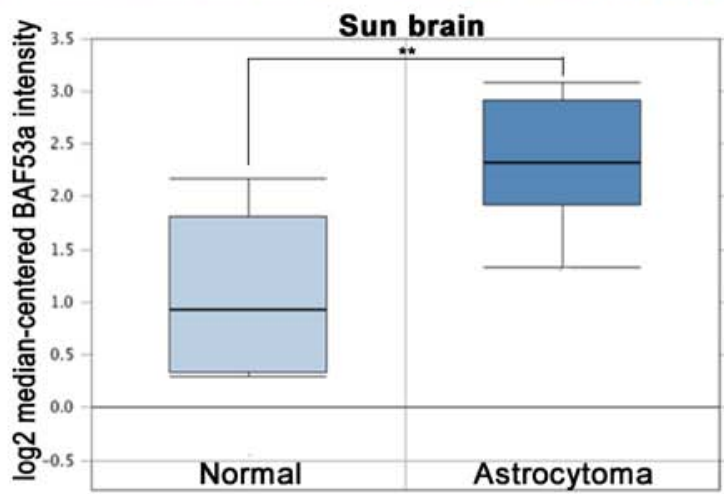

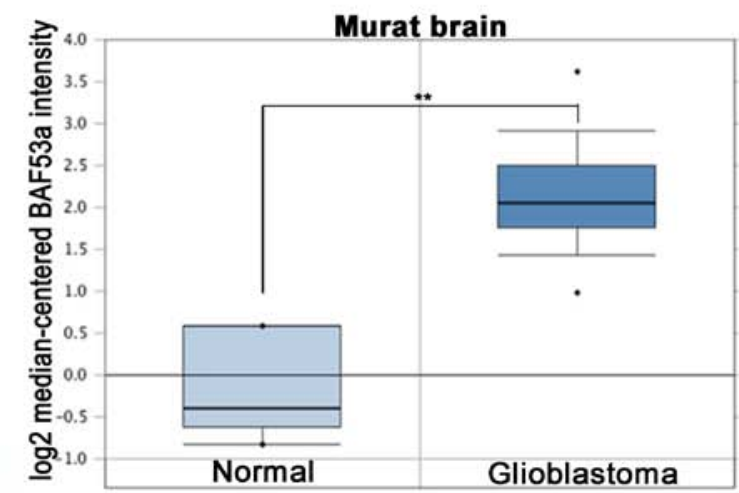

Figure 1. BAF53a expression is upregulated in glioma. (A) The representative images of BAF53a expression is detected by IHC in glioma tissues (scored as - to +++). Magnification, x400. (B) The real-time PCR results show that the expression level of BAF53a mRNA in glioma tissues was higher than that in normal brain tissues. The expression profile of BAF53a in Oncomine database: BAF53a gene copy number in astrocytoma is higher than normal brain tissues in Sun brain data (C), and BAF53a gene copy number in glioblastomas is significantly higher than normal brain tissues in TCGA brain data and Murat brain data (D). ${ }^{*} \mathrm{P}<0.05 ;{ }^{* *} \mathrm{P}<0.01$.

Migration and invasion assays. The migration potential was evaluated by wound-healing and Transwell assay. The cells were cultured into 6 -well plates at a density of $2 \times 10^{5}$ cells/well, when grew to $90 \%$ confluence, cells were incubated with mitomycin-C $(10 \mu \mathrm{g} / \mathrm{ml})$ for $1 \mathrm{~h}$ to suppress proliferation, and starved in serum-free medium for $24 \mathrm{~h}$. When cells were confluent monolayer artificial wound was scraped by a $10-\mu 1$ pipette tip. The migration gap of the wound was assessed after 24 or $48 \mathrm{~h}$. The migration potential was evaluated by Transwell system. The cells treated with mitomycin-C $(10 \mu \mathrm{g} / \mathrm{ml})$ for $1 \mathrm{~h}$ at $37^{\circ} \mathrm{C}$ in serum-free medium were placed into them with a density of $1 \times 10^{5}$ cells/insert. The DMEM with $10 \%$ FBS was added into the lower chamber. After incubation for 24-48 h, the cells in the upper membrane of insert were removed and the cells adhering to the lower membrane of the inserts were stained with crystal violet and counted by microscope after rinsing. Then the invasion potential was evaluated by Transwell-Matrigel system. The culture upper inserts were coated with Matrigel, the subsequence processes were carried out as Transwell assay. All the experiments were performed in triplicates.
Statistical analysis. Statistical analysis was conducted with SPSS version 18.0 (SPSS, Inc., Chicago, IL, USA). Student's two-tailed t-test was used to determine the statistical significance of measurement data. Survival curves were performed by the Kaplan-Meier method and compared by the log-rank test. Univariate and multivariate Cox regression model was used for survival analysis. The continuous data are shown as the mean \pm SEM. A P-value of $<0.05$ was considered statistically significant.

\section{Results}

BAF53a is highly expressed in glioma specimens. To examine the role of BAF53a in glioma, we initially detected the protein expression of BAF53a in tumor tissues by immunohistochemistry (IHC). BAF53a was predominantly expressed in the nucleus. We defined high BAF53a expression as strongly $(+++)$ or moderately $(++)$ positive staining, and low BAF53a expression as weakly positive $(+)$ or negative staining (Fig. 1A). BAF53a was positively expressed in the 

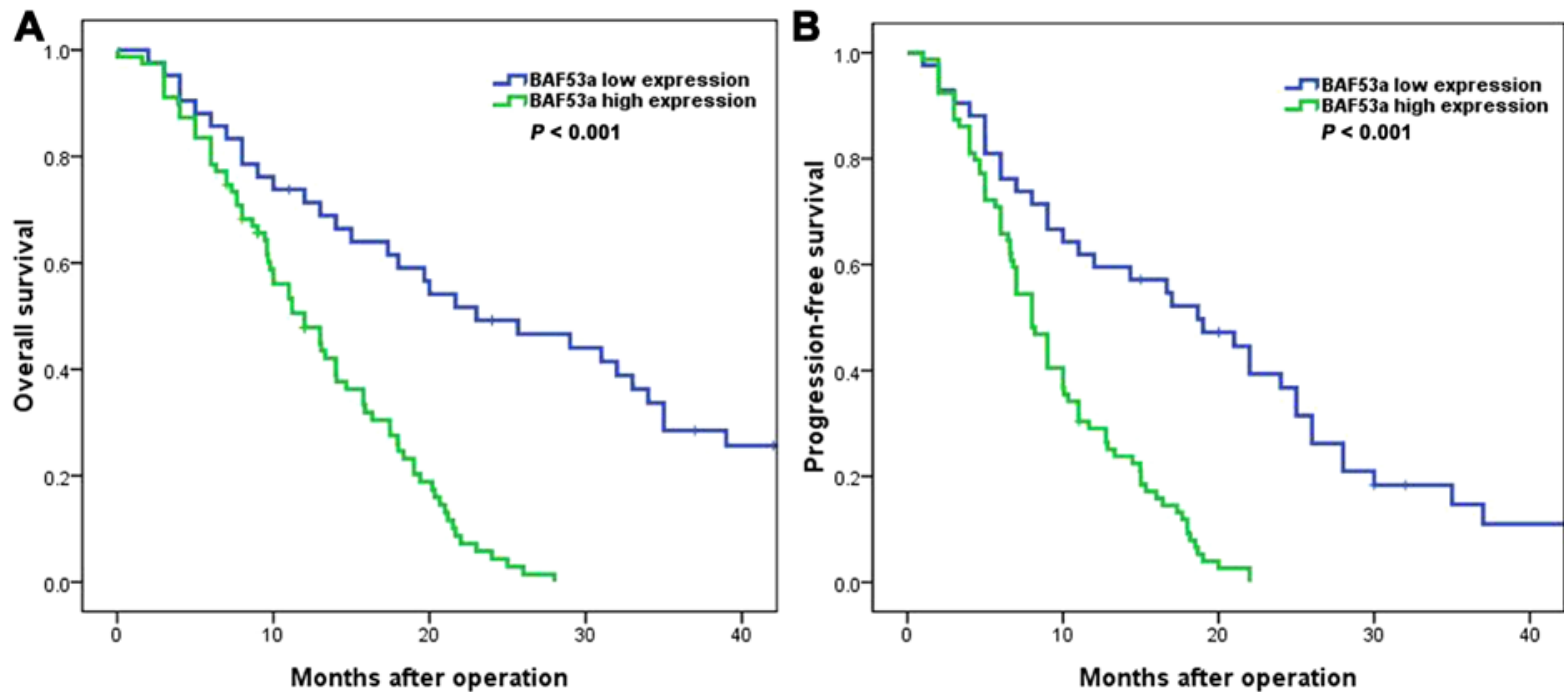

Figure 2. The survival curve of glioma patients analyzed according to the expression of BAF53a. (A) Results showed that glioma patients in the high BAF53a expression group had poorer overall survival than those in the low BAF53a expression group. (B) Glioma patients in the high BAF53a expression group had poorer progression-free survival than those in the low BAF53a expression group.

Table I. BAF53a expression and clinicopathologic features of 121 glioma cases.

\begin{tabular}{|c|c|c|c|c|}
\hline \multirow[b]{2}{*}{ Variables } & \multirow[b]{2}{*}{$\begin{array}{c}\text { Total } \\
\text { n (121) }\end{array}$} & \multicolumn{2}{|c|}{ BAF53a expression } & \\
\hline & & Low (42) & High (79) & P-value \\
\hline
\end{tabular}

Sex

$\begin{array}{lllll}\text { Female } & 53 & 17 & 36 & \\ \text { Male } & 68 & 25 & 43 & 0.591 \\ \text { Age (years) } & & & & \\ \leq 50 & 72 & 29 & 43 & \\ >50 & 49 & 13 & 36 & 0.119\end{array}$

Tumor size $(\mathrm{cm})$

$\begin{array}{lllll}\leq 4 & 66 & 25 & 41 & \\ >4 & 55 & 17 & 38 & 0.423\end{array}$

Necrosis

\begin{tabular}{lllll} 
Absence & 71 & 26 & 45 & \\
Presence & 50 & 16 & 34 & 0.599 \\
KPS score & & & & \\
$\leq 90$ & 82 & 31 & 51 & \\
$>90$ & 39 & 11 & 28 & 0.299 \\
WHO grade & & & & \\
I and II & 53 & 24 & 29 & \\
III and IV & 68 & 18 & 50 & 0.031 \\
\hline
\end{tabular}

KPS, Karnofsky performance status; WHO, World Health Organization.

majority of glioma tissues (102/121), of which 79 exhibited high expression (Table I). Additionally, BAF53a mRNA expression was examined in 10 randomly selected cases of glioma and 10 normal brain tissues obtained from resection following trauma. These results indicated that BAF53a mRNA expression levels in glioma tissues were significantly higher than those in normal brain tissues ( $\mathrm{P}<0.01$; Fig. 1B).

Subsequently, the expression profile of BAF53a was explored using the Oncomine ${ }^{\mathrm{TM}}$ database (https://www.oncomine.org). The results showed that the gene copy number of BAF53a in astrocytoma was higher than that in normal brain tissue (Sun brain: fold change, 2.460; P $<0.01$; Fig. 1C). Furthermore, the BAF53a gene copy number in glioblastoma was significantly higher than that in normal brain tissue (TCGA brain: fold change, 11.006; $\mathrm{P}<0.01$; Murat brain: fold change, 5.298; $\mathrm{P}=0.002$; Fig. 1D). These results enable us to speculate that BAF53 may be involved in the progression of glioma.

BAF53a expression is associated with poor prognosis in patients with glioma. We next explored the detailed associations between BAF53a protein expression (determined by IHC) and the clinicopathological features and prognosis of glioma patients. The results indicated that high BAF53a expression was significantly correlated with WHO grade $(\mathrm{P}=0.031$; Table I). Survival analysis showed that the BAF53a low expression group had favorable overall survival (OS) [median OS time, 23.0 (95\% CI, 11.6-34.4) vs. 12.0 (95\% CI, 8.9-15.1) months; $\mathrm{P}<0.001$; Fig. 2A] and progression-free survival (PFS) [median PFS time, 18.7 (95\% CI, 10.6-26.8) vs. 8.0 (95\% CI, 6.7-9.3) months, $\mathrm{P}<0.001$; Fig. 2B] compared with the BAF53a high expression group. Furthermore, univariate and multivariate Cox regression analyses indicated that BAF53a expression was an independent prognostic factor for the OS and PFS of glioma patients $(\mathrm{HR}=1.752, \mathrm{P}=0.013$ and $\mathrm{HR}=1.874, \mathrm{P} 0.008$, respectively; Tables II and III). These results indicate that BAF53a may be useful as a prognostic marker for patients with glioma.

BAF53 a promotes the proliferation of glioma cells in vitro. In order to study the functional role of BAF53a in glioma, stable BAF53a-overexpressing U87 ${ }^{\text {BAF53a }}$ and BAF53a-knockdown U87 ${ }^{\text {shBAF53a }}$ cells and control cells transfected with empty vector 
Table II. The univariate and multiple Cox regression analyses of overall survival (OS) in glioma patients.

\begin{tabular}{|c|c|c|c|c|}
\hline \multirow[b]{2}{*}{ Variables } & \multicolumn{2}{|c|}{ Univariable analysis } & \multicolumn{2}{|c|}{ Multivariable analysis } \\
\hline & $\mathrm{HR}(95 \% \mathrm{CI})$ & P-value & $\mathrm{HR}(95 \% \mathrm{CI})$ & P-value \\
\hline Sex (male vs. female) & $1.322(0.492-2.485)$ & 0.251 & & \\
\hline Age ( $\leq 50$ vs. $>50$ years) & $1.183(0.642-1.934)$ & 0.426 & & \\
\hline Tumor size $(\leq 4$ vs. $>4 \mathrm{~cm})$ & $0.613(0.382-1.267)$ & 0.137 & & \\
\hline Necrosis (absence vs. presence) & $0.714(0.433-1.162)$ & 0.325 & & \\
\hline KPS score ( $\leq 90$ vs. $>90)$ & $0.682(0.310-1.254)$ & 0.185 & & \\
\hline WHO grade (III and IV vs. I and II) & $3.126(1.643-5.233)$ & $<0.001$ & $2.304(1.192-3.542)$ & 0.002 \\
\hline BAF53a expression (high vs. low) & $1.937(1.326-3.174)$ & 0.006 & $1.752(1.226-2.638)$ & 0.013 \\
\hline
\end{tabular}

HR, hazard risk ratio; CI, confidence interval. Bold, statistically significant.

Table III. The univariate and multiple Cox regression analyses of progression-free survival (PFS) in glioma patients.

\begin{tabular}{|c|c|c|c|c|}
\hline \multirow[b]{2}{*}{ Variables } & \multicolumn{2}{|c|}{ Univariable analysis } & \multicolumn{2}{|c|}{ Multivariable analysis } \\
\hline & $\mathrm{HR}(95 \% \mathrm{CI})$ & P-value & $\mathrm{HR}(95 \% \mathrm{CI})$ & P-value \\
\hline Sex (male vs. female) & $1.167(0.643-1.823)$ & 0.572 & & \\
\hline Age ( $\leq 50$ vs. $>50$ years) & $1.094(0.735-1.541)$ & 0.714 & & \\
\hline Tumor size ( $\leq 4$ vs. $>4 \mathrm{~cm})$ & $0.582(0.221-1.142)$ & 0.095 & $0.623(0.334-1.095)$ & 0.142 \\
\hline Necrosis (absence vs. presence) & $0.802(0.455-1.372)$ & 0.483 & & \\
\hline KPS score $(\leq 90$ vs. $>90)$ & $0.713(0.386-1.197)$ & 0.224 & & \\
\hline WHO grade (III and IV vs. I and II) & $4.075(1.825-7.437)$ & $<0.001$ & $3.194(1.426-5.273)$ & $<0.001$ \\
\hline BAF53a expression (high vs. low) & $2.056(1.274-3.312)$ & 0.003 & $1.874(1.411-2.942)$ & 0.008 \\
\hline
\end{tabular}

were established. The transfection efficiency in each cell type was confirmed by qPCR and western blotting (Fig. 3A and B). Subsequently, the cells were subjected to MTT and colony formation assays. The MTT assay revealed that BAF53a overexpression could markedly increase U87 cell growth, while BAF53a knockdown had the opposite effect as compared with control cells $(\mathrm{P}<0.05$, respectively; Fig. $3 \mathrm{C})$. The colony formation assay also showed that the U87 ${ }^{\text {BAF53a }}$ cells were significantly larger and greater in number than the control cells, while U87 ${ }^{\text {shBAF53a }}$ cells exhibited the opposite characteristics $(\mathrm{P}<0.05$, respectively; Fig. 3D). These results indicated that BAF53a could promote the proliferation of glioma cells.

BAF53a promotes the migration and invasion of glioma cells in vitro. Wound healing and Transwell assays were used to assess the migratory ability of U87 cells expressing varying levels of BAF53a. The results showed that BAF53a-overexpressing U87 ${ }^{\text {BAF53a }}$ cells exhibited increased migration and faster wound healing capacities, whereas U87 ${ }^{\text {shBAF53a }}$ cells exhibited decreased migration and slower wound healing capacities when compared with control cells (Fig. 3E and F). A Transwell-Matrigel chamber assay accordingly showed that $U 87^{\text {BAF53a }}$ cells had a greater capacity for invasion across the Matrigel membrane compared with the control cells, whereas U87 $7^{\text {shBAF53a }}$ cells had reduced invasive ability (Fig. 3G). These results indicated that BAF53a could promote the invasion capacity of glioma cells and potentially facilitate the metastasis of glioma.

BAF53a expression is associated with EMT in glioma. As previous studies have reported a possible link between BAF53a and EMT in other physiological processes and cancer progression, we aimed to explore this association in glioma. The expression levels of two EMT markers, E-cadherin and vimentin, were detected in cells expressing different levels of BAF53a. The qPCR and western blotting results showed decreased expression of the epithelial marker E-cadherin and increased expression of the mesenchymal marker vimentin in U87 ${ }^{\text {BAF53a }}$ cells. By contrast, the opposite pattern of expression was demonstrated in U87shBAF53a cells (Fig. 4A and B). Alterations in cellular morphology assessed by phase contrast microscopy also revealed that U87 ${ }^{\text {BAF53a }}$ cells had more pseudopodia and were elongated, indicating a mesenchymal phenotype, whereas U87 ${ }^{\text {shBAF53a }}$ cells had an oval or cobblestone-like appearance (Fig. 4C). E-cadherin and vimentin expression were also detected in glioma tissues by IHC. The results revealed that E-cadherin expression was frequently absent in glioma, whereas vimentin was consistently expressed (Fig. 4D). The Spearman rank correlation coefficient indicated that BAF53a expression was positively 

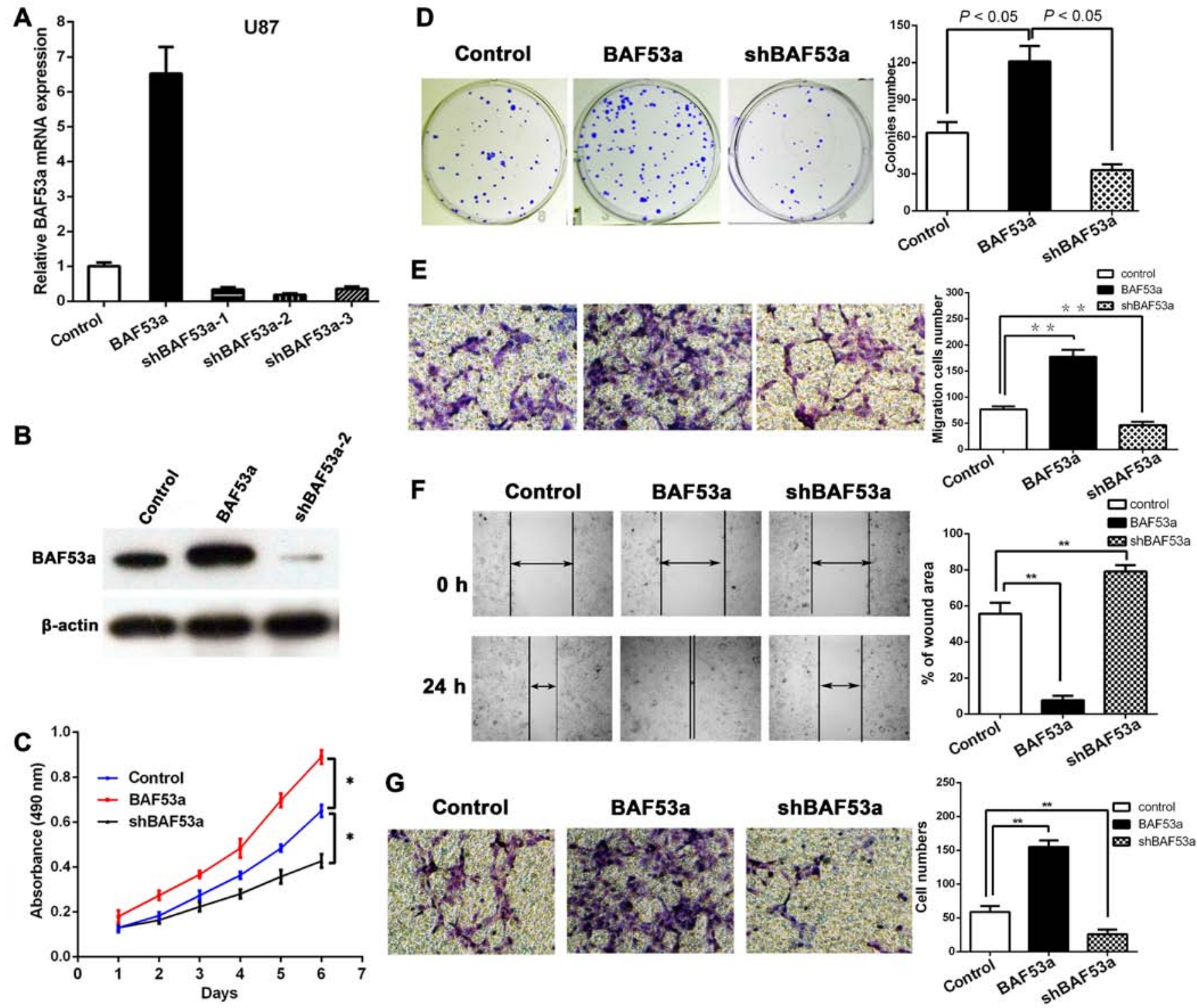

Figure 3. BAF53a promotes proliferation, migration and invasion of glioma cells. The overexpression and knockdown interfered efficiency of BAF53a detected by real-time PCR (A) and western blotting (B), results showed shBAF53a-2 had the best knockdown effect and the overexpression effect was also satisfactory. (C) The MTT assays showed BAF53a overexpression promoted glioma cell proliferation, while BAF53a knockdown inhibited glioma cell proliferation. (D) The colony formation assays also showed BAF53a expression promoted glioma cell proliferation. (E) The Transwell assay showed BAF53a expression facilitated migration of glioma cells. (F) The wound healing assay showed BAF53a expression provided the faster wound healing capacities of glioma cells. (G) The Transwell-Matrigel chamber assay showed BAF53a expression facilitated invasion of glioma cells.

correlated with the expression of vimentin $(\mathrm{r}=0.384, \mathrm{P}<0.001)$ and negatively correlated with the expression of E-cadherin $(\mathrm{r}=-0.367, \mathrm{P}<0.001$ ) (Fig. 4E). Taken together, these results suggest that BAF53a may promote glioma progression via EMT.

\section{Discussion}

The findings of the present study revealed that BAF53a is a potential valuable prognostic marker for glioma patients, and that it may promote glioma cell proliferation, migration and invasion and be associated with EMT.

Notably, the present study demonstrated the functional role of BAF53a in promoting glioma progression. The subunits of the BAF complex are known to serve important roles in many physiological and pathological processes in various cell types and organs. At present, the association of BAF53a with cancer development and progression is attracting increasing attention. In 2002, a study demonstrated that BAF53a was critical for the oncogenic activity of c-Myc (17). Additionally, studies of rhabdomyosarcoma, hepatocellular carcinoma and squamous cell carcinoma have also provided evidence that BAF53a is essential for cancer invasion and metastasis, and is associated with poor prognosis $(13,15,16)$. Studies have also demonstrated that the subunits of the BAF complex, including ARID2, ARID5B and BAF47, play important roles in a variety of cancer types (18-20). These results are consistent with those of the present study of glioma, which showed that BAF53a may be a cancer-promoting gene.

According to previous studies, BAF53a knockdown can significantly impair neural stem/progenitor cell proliferation capacity and their differentiation into neurons $(12,21)$. Another study showed that BAF53a was overexpressed in fibroblasts and blocked the neuronal conversion of fibroblasts (22). A 
A

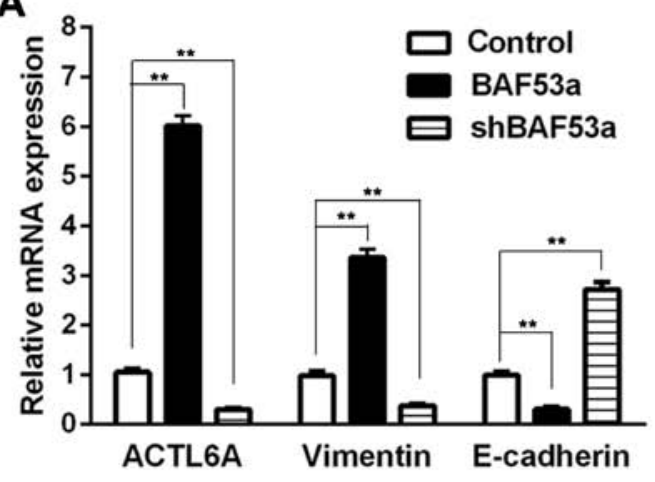

B

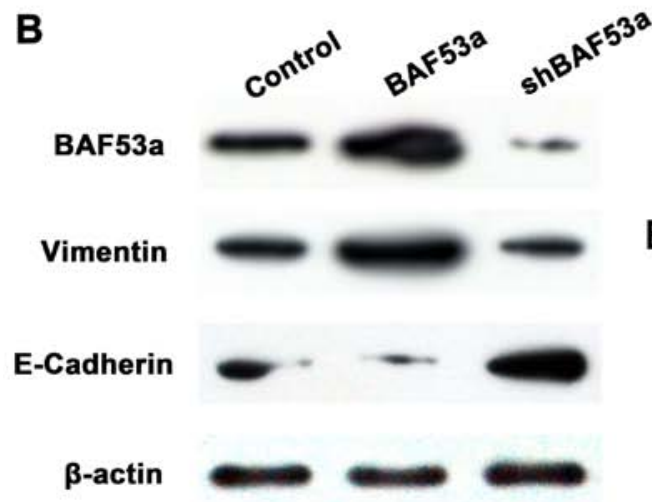

C Control

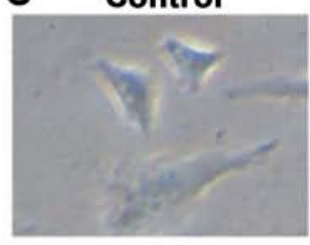

D

BAF53a
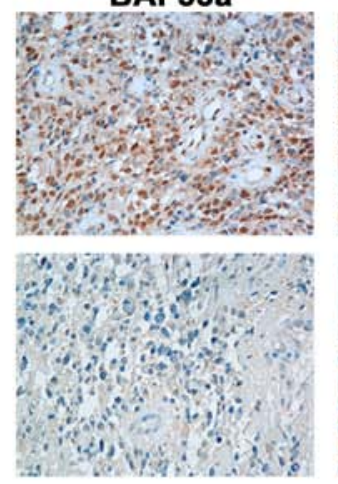

E

\begin{tabular}{|c|c|c|c|c|c|c|}
\hline & $\begin{array}{r}V_{i} \\
\text { ex } \\
\operatorname{High}(\theta\end{array}$ & $\begin{array}{l}\text { tin } \\
\text { sion } \\
\text {-ow(52) }\end{array}$ & $\begin{array}{l}P \\
r\end{array}$ & $\begin{array}{l}\text { E-ck } \\
\text { exp } \\
\text { High }(3\end{array}$ & $\begin{array}{l}\text { erin } \\
\text { sion } \\
\text { sow(90) }\end{array}$ & $\begin{array}{l}P \\
r\end{array}$ \\
\hline \multirow{2}{*}{$\begin{array}{l}\text { BAF53a High (79) } \\
\text { expression Low (42) }\end{array}$} & ) 56 & 23 & $<0.001$ & 11 & 68 & $<0.001$ \\
\hline & ) 13 & 29 & 0.384 & 20 & 22 & -0.367 \\
\hline
\end{tabular}

Figure 4. BAF53a associates with EMT in glioma. The expression correlation of BAF53a, vimentin and E-cadherin in glioma cells detected by real-time PCR (A) and western blotting (B), results showed that vimentin expression was upregulated along with BAF53a overexpression, but E-cadherin expression was decreased; when BAF53a was knocked down, they had inverse expression. (C) The phase contrast images of BAF53a-interfered U87 cells, results showed cells became mesenchymal-like in BAF53a overexpression, and cells changed to oval or cobblestone-like appearance in BAF53a knockdown. (D) The representative images of expression correlation of BAF53a, vimentin and E-cadherin in glioma tissues detected by IHC. (E) The Spearman's rank correlation test indicated that BAF53a and vimentin expression were positively correlated, while BAF53a and E-cadherin expression were negatively correlated.

study by Krasteva et al also found that the deletion of BAF53a resulted in embryonic lethality and proliferation defects of hematopoietic stem cells (11). Furthermore, a recent study by Bao et al showed that ectopic expression of BAF53a promoted the proliferation and progenitor state of epidermal cells (23). These studies suggest that BAF53a can control differentiation and proliferation in embryonic and adult stem cells, as well as during embryogenesis. It is well established that cancer progression shares many similar events with embryogenesis, such as cell growth, differentiation and migration. Therefore, genes that are crucial for embryonic or stem cell development are often associated with cancer progression. Accordingly, the present study has verified the hypothesis that BAF53a can promote glioma progression and lead to poor prognosis.

When cancer cells undergo EMT, they acquire increased motility and invasive capacity, as well as resistance to chemoradiotherapy; EMT thereby promotes metastasis and results in poor prognosis. In glioma, various genes and non-coding RNAs, such as AC1MMRY2, MLK4 and miR$200 \mathrm{a} / \mathrm{b}$, are reportedly able to induce EMT, particularly in glioblastoma $(24,25)$. Notably, the present data indicated that alteration of BAF53a expression could induce morphological changes in glioma cells, as well as influencing the expression levels of epithelial and mesenchymal markers. This suggested that BAF53a may induce EMT in glioma and function as an
EMT-related transcriptional factor. These results illustrate a novel biological function of BAF53a in glioma, which is consistent with previous studies of osteosarcoma and HCC $(14,16)$. Additionally, a recent study in HNSCC found that BAF53a could activate YAP1, which can induce EMT in various cancer types, and these findings confirm the role of BAF53a in EMT $(13,26)$.

EMT inhibitors, such as BGB324 and A83-01, are designed to target receptors in the cytomembrane; however, targeting EMT-related genes in the cell nucleus may be a more precise approach. Therefore, the present results provide the basis to potentially develop a novel targeted therapy.

The mechanism by which BAF53a induces EMT remains unclear. Previous studies found that BAF53a could interact with p63, SALL4, FGF4 and KLF4 to maintain the stemness and undifferentiated state of stem/progenitor cells, which is important for the acquisition of mesenchymal characteristics $(10,13,23)$. A study of HCC showed that BAF53a could regulate the transcription of SOX2 to further activate the Notch pathway to induce EMT (16). However, the detailed molecular mechanism regarding the contribution of BAF53a to EMT in glioma still requires further research.

The present study had certain limitations. First, the BAF53a expression profile and its prognostic significance were only retrospectively analyzed in glioma tissues and in a 
single institution; this need to be expanded to different cancer types and validated in an external cohort in the future. Second, the association between BAF53 and EMT requires further in vitro and in vivo experiments to support the hypothesis. Third, further research into the precise molecular mechanisms of BAF53 in promoting glioma progression and EMT is necessary.

In conclusion, the present study suggests that high BAF53a expression predicts poor prognosis of glioma patients, and that BAF53a can promote invasion and EMT of glioma cells. Therefore, BAF53a may be a useful prognostic marker and potential promising target for glioma therapeutics.

\section{References}

1. Wen PYKS and Kesari S: Malignant gliomas in adults. N Engl J Med 359: 492-507, 2008.

2. Chen R, Smith-Cohn M, Cohen AL and Colman H: Glioma subclassifications and their clinical significance. Neurotherapeutics 14: 284-297, 2017.

3. Zhang X, Yang H, Gong B, Jiang C and Yang L: Combined gene expression and protein interaction analysis of dynamic modularity in glioma prognosis. J Neurooncol 107: 281-288, 2012.

4. Alderton GK: Metastasis: Epithelial to mesenchymal and back again. Nat Rev Cancer 13: 3, 2013.

5. Hanahan D and Weinberg RA: Hallmarks of cancer: The next generation. Cell 144: 646-674, 2011.

6. Thiery JP, Acloque H, Huang RY and Nieto MA: Epithelialmesenchymal transitions in development and disease. Cell 139: 871-890, 2009.

7. Kahlert UD, Maciaczyk D, Doostkam S, Orr BA, Simons B, Bogiel T, Reithmeier T, Prinz M, Schubert J, Niedermann G, et al: Activation of canonical WNT/ $\beta$-catenin signaling enhances in vitro motility of glioblastoma cells by activation of ZEB1 and other activators of epithelial-to-mesenchymal transition. Cancer Lett 325: 42-53, 2012.

8. Zhang L, Zhang W, Li Y, Alvarez A, Li Z, Wang Y, Song L, Lv D, Nakano I, Hu B, et al: SHP-2-upregulated ZEB1 is important for PDGFR $\alpha$-driven glioma epithelial-mesenchymal transition and invasion in mice and humans. Oncogene 35: 5641-5652, 2016.

9. Kahlert UD, Nikkhah G and Maciaczyk J: Epithelial-tomesenchymal(-like) transition as a relevant molecular event in malignant gliomas. Cancer Lett 331: 131-138, 2013.

10. Lu W, Fang L, Ouyang B, Zhang X, Zhan S, Feng X, Bai Y, Han X, Kim H, He Q, et al: Actl6a protects embryonic stem cells from differentiating into primitive endoderm. Stem Cells 33: 1782-1793, 2015.

11. Krasteva V, Buscarlet M, Diaz-Tellez A, Bernard MA, Crabtree GR and Lessard JA: The BAF53a subunit of SWI/ SNF-like BAF complexes is essential for hemopoietic stem cell function. Blood 120: 4720-4732, 2012.

12. Lessard J, Wu JI, Ranish JA, Wan M, Winslow MM, Staahl BT, Wu H, Aebersold R, Graef IA and Crabtree GR: An essential switch in subunit composition of a chromatin remodeling complex during neural development. Neuron 55: 201-215, 2007.
13. Saladi SV, Ross K, Karaayvaz M, Tata PR, Mou H, Rajagopal J, Ramaswamy S and Ellisen LW: ACTL6A is co-amplified with p63 in squamous cell carcinoma to drive YAP activation, regenerative proliferation, and poor prognosis. Cancer Cell 31: 35-49, 2017.

14. Sun W, Wang W, Lei J, Li H and Wu Y: Actin-like protein 6A is a novel prognostic indicator promoting invasion and metastasis in osteosarcoma. Oncol Rep 37: 2405-2417, 2017.

15. Taulli R, Foglizzo V, Morena D, Coda DM, Ala U, Bersani F, Maestro N and Ponzetto C: Failure to downregulate the BAF53a subunit of the SWI/SNF chromatin remodeling complex contributes to the differentiation block in rhabdomyosarcoma. Oncogene 33: 2354-2362, 2014

16. Xiao S, Chang RM, Yang MY, Lei X, Liu X, Gao WB, Xiao JL and Yang LY: Actin-like 6A predicts poor prognosis of hepatocellular carcinoma and promotes metastasis and epithelial-mesenchymal transition. Hepatology 63: 1256-1271, 2016.

17. Park J, Wood MA and Cole MD: BAF53 forms distinct nuclear complexes and functions as a critical c-Myc-interacting nuclear cofactor for oncogenic transformation. Mol Cell Biol 22: 1307-1316, 2002.

18. Li M, Zhao H, Zhang X, Wood LD, Anders RA, Choti MA, Pawlik TM, Daniel HD, Kannangai R, Offerhaus GJ, et al: Inactivating mutations of the chromatin remodeling gene ARID2 in hepatocellular carcinoma. Nat Genet 43: 828-829, 2011.

19. Kandoth C, Schultz N, Cherniack AD, Akbani R, Liu Y, Shen H, Robertson AG, Pashtan I, Shen R, Benz CC, et al; Cancer Genome Atlas Research Network: Integrated genomic characterization of endometrial carcinoma. Nature 497: 67-73, 2013.

20. Kadoch C and Crabtree GR: Reversible disruption of mSWI/ SNF (BAF) complexes by the SS18-SSX oncogenic fusion in synovial sarcoma. Cell 153: 71-85, 2013.

21. Yoo AS, Staahl BT, Chen L and Crabtree GR: MicroRNAmediated switching of chromatin-remodelling complexes in neural development. Nature 460: 642-646, 2009.

22. Yoo AS, Sun AX, Li L, Shcheglovitov A, Portmann T, Li Y, Lee-Messer C, Dolmetsch RE, Tsien RW and Crabtree GR: MicroRNA-mediated conversion of human fibroblasts to neurons. Nature 476: 228-231, 2011.

23. Bao X, Tang J, Lopez-Pajares V, Tao S, Qu K, Crabtree GR and Khavari PA: ACTL6a enforces the epidermal progenitor state by suppressing SWI/SNF-dependent induction of KLF4. Cell Stem Cell 12: 193-203, 2013.

24. Kim SH, Ezhilarasan R, Phillips E, Gallego-Perez D, Sparks A, Taylor D, Ladner K, Furuta T, Sabit H, Chhipa R, et al: Serine/ threonine kinase MLK4 determines mesenchymal identity in glioma stem cells in an NF- $\kappa \mathrm{B}-$ dependent manner. Cancer Cell 29: 201-213, 2016.

25. Shi Z, Zhang J, Qian X, Han L, Zhang K, Chen L, Liu J, Ren Y, Yang M, Zhang A, et al: AC1MMYR2, an inhibitor of dicer-mediated biogenesis of Oncomir miR-21, reverses epithelial-mesenchymal transition and suppresses tumor growth and progression. Cancer Res 73: 5519-5531, 2013.

26. Shao DD, Xue W, Krall EB, Bhutkar A, Piccioni F, Wang X, Schinzel AC, Sood S, Rosenbluh J, Kim JW, et al: KRAS and YAP1 converge to regulate EMT and tumor survival. Cell 158: 171-184, 2014. 